\title{
Unsupervised classification of snowflake images using a generative adversarial network and $\boldsymbol{K}$-medoids classification
}

\author{
Jussi Leinonen and Alexis Berne \\ Environmental Remote Sensing Laboratory, École polytechnique fédérale de Lausanne, Lausanne, Switzerland
}

Correspondence: Jussi Leinonen (jussi.leinonen@epfl.ch)

Received: 18 November 2019 - Discussion started: 11 December 2019

Revised: 15 April 2020 - Accepted: 30 April 2020 - Published: 5 June 2020

\begin{abstract}
The increasing availability of sensors imaging cloud and precipitation particles, like the Multi-Angle Snowflake Camera (MASC), has resulted in datasets comprising millions of images of falling snowflakes. Automated classification is required for effective analysis of such large datasets. While supervised classification methods have been developed for this purpose in recent years, their ability to generalize is limited by the representativeness of their labeled training datasets, which are affected by the subjective judgment of the expert and require significant manual effort to derive. An alternative is unsupervised classification, which seeks to divide a dataset into distinct classes without expert-provided labels. In this paper, we introduce an unsupervised classification scheme based on a generative adversarial network (GAN) that learns to extract the key features from the snowflake images. Each image is then associated with a distribution of points in the feature space, and these distributions are used as the basis of $K$-medoids classification and hierarchical clustering. We found that the classification scheme is able to separate the dataset into distinct classes, each characterized by a particular size, shape and texture of the snowflake image, providing signatures of the microphysical properties of the snowflakes. This finding is supported by a comparison of the results to an existing supervised scheme. Although training the GAN is computationally intensive, the classification process proceeds directly from images to classes with minimal human intervention and therefore can be repeated for other MASC datasets with minor manual effort. As the algorithm is not specific to snowflakes, we also expect this approach to be relevant to other applications.
\end{abstract}

\section{Introduction}

The microphysical properties of atmospheric ice and snow have significant implications for several topics in atmospheric science. In numerical weather prediction (NWP) and climate models, the representation of ice processes has a considerable influence on the forecast (Molthan and Colle, 2012; Morrison et al., 2015; Gultepe et al., 2017; Elsaesser et al., 2017), affecting the distribution of predicted precipitation, latent heat and radiative effects. More generally, precipitation and clouds are recognized as being among the largest uncertainties in climate predictions (e.g., Flato et al., 2013). In another context, microphysical assumptions also play an important role in the remote sensing of ice and snow because the remotely obtained signal only conveys partial information about the properties of the icy hydrometeors, and retrieval algorithms need to be constrained by prior knowledge about the microphysics (e.g., Delanoë and Hogan, 2010; Wood et al., 2014; Mace and Benson, 2017; Leinonen et al., 2018).

Given the importance of microphysics, the observational geoscience community has made considerable efforts to develop instruments that characterize the microphysical properties of falling snowflakes in situ. Measuring the properties of individual falling snowflakes is fairly challenging, as some important properties such as the snowflake mass are not readily observable using visual techniques due to the variation of the internal structure of snowflakes. Moreover, the widespread optical disdrometers, such as the Parsivel (Löffler-Mang and Joss, 2000) and the 2D Video Disdrometer (2DVD; Schönhuber et al., 2007), can only discern a silhouette of the falling particle, unable to provide information about the surface texture. To address this issue, snowflake-imaging instruments have been actively developed 
in recent years. Among these is the Multi-Angle Snowflake Camera (MASC; Garrett et al., 2012), which employs three cameras positioned at different angles to captures images of snowflakes illuminated by a flash as they fall through its measurement volume. Other similar instruments include the Snowflake Video Imager (SVI; Newman et al., 2009, also known as Particle Video Imager or Particle Imaging Package) and the Dual Ice Crystal Imager (D-ICI; Kuhn and VázquezMartín, 2020).

The datasets collected so far by various groups (e.g., Gaustad et al., 2015; Notaroš et al., 2016; Praz et al., 2017; Genthon et al., 2018) show that the detailed images obtained by the MASC provide a signature of the processes that led to the formation of each snowflake. The MASC can discern processes such as various modes of deposition growth like columns, plates and dendrites, as well as aggregation, riming and melting (for an overview of these, see, e.g., Lamb and Verlinde, 2011). As these processes depend on the environmental conditions in which the snowflake grew, the MASC can provide information about the relative occurrence of these conditions in a specific snowfall event and, over longer timescales, the local climate.

While trained human observers can determine the presence of various snow growth processes from MASC images, the large datasets collected by the MASC require computer analysis in order to derive statistically meaningful quantities of data. The computer processing of the image data is not straightforward because much of the information is provided by shape and the surface texture of the snowflake. Feind (2006) and Lindqvist et al. (2012), among others, previously developed algorithms to classify ice crystals based on images from airborne probes. To enable large-scale analysis of microphysics from MASC data, Praz et al. (2017, hereafter P17) introduced a machine-learning-based classification algorithm that uses features extracted from the images with image-processing software, providing information about the size, shape and surface patterns of each snowflake. This algorithm can classify the snowflakes and also estimate the degree of riming and the state of melting, enabling microphysical information to be extracted on long timescales.

The development of convolutional neural networks (CNNs) has recently greatly improved the image-recognition skill of computers. Deep CNNs, which consist of a large number of successive convolution layers and nonlinearities, have proved to be able to classify images based on the image data alone, without manual feature extraction. Instead, the CNN adaptively learns the important features of images that it is trained with. Such advances could be reasonably expected to lend themselves well to MASC data analysis, and indeed Hicks and Notaroš (2019) recently described a CNNbased classification scheme for MASC images that achieved a performance comparable to the P17 algorithm.

Supervised learning methods, such as those adopted by the abovementioned studies, depend on the availability of an expert-derived training dataset to train them. Obtaining such datasets is labor intensive, especially for CNNs that benefit from large amounts of training data. Moreover, developing the training datasets is somewhat subjective as it depends on the judgment of the expert to determine the "correct" classification for each image. The alternative is unsupervised classification, which tries to organize the training data without human intervention. Unsupervised classification methods are able to operate on entire datasets without training labels, and can be less subjective than supervised classification, but are also more complex and difficult to implement as the role of the computer-based learning system in the process is much larger. Furthermore, unsupervised classification of images requires the extraction of those features of the images that are essential to classification, and ideally those features should themselves be determined in an unsupervised manner.

Unsupervised learning from image data has recently benefited from the introduction of generative adversarial networks (GANs; Goodfellow et al., 2014). GANs consist of two neural networks (usually deep CNNs), the discriminator and the generator. These are trained adversarially: the discriminator is trained to distinguish samples that belong to the training dataset from those that do not, while the generator is trained to produce samples that the discriminator considers to be part of the training data. Consequently, the generator learns to create artificial samples that strongly resemble those found in the dataset. GANs have been recently demonstrated to be able to create realistic examples of, for example, human faces (Karras et al., 2019) and landscapes (Park et al., 2019), and have been demonstrated to be applicable to atmospheric science data analysis (Leinonen et al., 2019).

The GAN generator is a deterministic neural network, but it can produce different outputs because it is fed random noise as an input. The random noise is sampled from a simple probability distribution such as the multivariate standard normal distribution. Thus, the generator learns to map the simple probability distribution to the highly complex, spatially structured distribution of the image dataset. It is fairly straightforward to add another output to the discriminator, which is trained to recover all or part of the noise input of the generator, as demonstrated by the GAN variant called the Information-Maximizing GAN (InfoGAN; Chen et al., 2016). Accordingly, the recovered part of the noise input can be understood as latent variables that encode certain learned properties of the images, and thus the generator acts a decoder from the latent variables to image samples, and the discriminator as an encoder with the approximately inverse map. The training process encourages both the generator and the discriminator to map the latent variables to highly recognizable features of the images, thus capturing their essential properties. In the original InfoGAN paper, it was shown that InfoGAN can, in an unsupervised manner, recognize important modes of variation in its input images. Similar results have been achieved with a related GAN variant called the bidirectional GAN (Donahue et al., 2017). 
In this article, we describe a GAN trained on a dataset of MASC images and the use of the GAN-extracted latent variables for unsupervised classification with the $K$-medoids algorithm. We show that a more consistent classification can be achieved by associating each image with a distribution of points rather than a single point in the latent space. This combination of a GAN and a more traditional unsupervised machine learning algorithm can be used to classify snowflake datasets without human intervention.

The article is organized as follows. Section 2 describes the snowflake datasets and data processing. Section 3 gives an overview of the machine learning methodology, and Sect. 4 describes the implementation details used for this work. Section 5 discusses the classification results for the snowflake data and presents a quantitative and qualitative evaluation of them. Finally, Sect. 6 summarizes the study.

\section{Data}

The main source of our snowflake image dataset is the deployment of a MASC in Davos, Switzerland, during 2015 and 2016. The MASC was located at $2450 \mathrm{~m}$ above mean sea level, with a long snowy season, and was enclosed by a Double Fence Intercomparison Reference (DFIR) setup. The dataset includes a wide variety of snowflakes, including single crystals of different morphologies, aggregates, rimed snowflakes and graupel, as well as partially melted snowflakes. Additionally, in order to increase the diversity of the dataset we used data from the Antarctic Precipitation, Remote Sensing from Surface and Space (APRES3) campaign (Genthon et al., 2018), where a MASC was deployed in Dumont D'Urville on the coast of East Antarctica. We concentrated on this joint dataset in this study, as we did not find the results to be very different from a classification trained only on the Davos dataset.

We first filtered out blowing snow particles from the data from APRES3, where the MASC was not shielded by a wind fence, using the results of Schaer et al. (2020). The raw data were then processed using the processing chain described by $\mathrm{P} 17$, which crops the original MASC images into rectangular areas containing only the snowflake; rejects unsuitable candidates, such as those intersecting with the edge of the MASC image; and computes various image quality metrics. The resulting dataset includes approximately 2.1 million grayscale snowflake images, 1.9 million from Davos and 0.2 million from APRES3. From these, we selected high-quality images as follows. We selected snowflakes whose outlines were between 16 and 256 pixels in diameter; with the MASC resolution of approximately $35 \mu \mathrm{m}$ per pixel, this corresponds to a physical size roughly between 0.5 and $9 \mathrm{~mm}$. The lower limit was chosen to filter out occasional artifacts and snowflakes too small to be recognized; the rarely applied upper limit was used to ensure that each snowflake fits entirely in the image. We also removed all particles classified as "small par- ticles" by the P17 algorithm, although not many of these remained after imposing the minimum size. To filter out blurry snowflakes, we required that the quality index $\xi$, defined in Appendix B of P17, be at least 10 . The minimum $\xi$ is higher than in P17 because we wanted to avoid training the GAN to generate blurry images. Furthermore, to remove snowflakes that were not bright enough, as well as some artifacts that passed the $\xi$ test, we required that the brightest pixel of the image be at least 0.15 on a scale of 0 to 1 . The final dataset used for training comprises 195006 snowflake images, 166981 from Davos and 28085 from APRES3.

The snowflake images were downsampled by a factor of 2 in order to reduce the computational burden; this could be done without losing much information because MASC images are usually at least slightly blurry and therefore the true resolution of the images is not quite as good as the pixel resolution. Each image was then centered into a $128 \times 128$ pixel frame in order to yield constant-sized images, as needed for training. Many images included fairly dim areas; to make these more visible, we applied the following transformation to the brightness:

$f(x)= \begin{cases}\frac{b}{a} x, & x<a \\ b+\frac{1-b}{1-a}(x-a), & x \geq a,\end{cases}$

with $a=0.1$ and $b=0.2$ chosen, somewhat subjectively, to improve the visibility of dark snowflakes without losing too much contrast. This maps brightness values $0 \rightarrow 0, a \rightarrow b$ and $1 \rightarrow 1$ and linearly between these points. The resulting image dataset has a mean brightness of 0.28 for non-empty pixels (i.e., those of brightness $>0$ ).

Following standard practice in training convolutional neural networks, the sample diversity was increased during training using data augmentation. Before using images for training, the following random augmentations were performed to each image:

- rotation by an angle between 0 and $360^{\circ}$;

- mirroring around the vertical and/or horizontal axes;

- zooming the image by a factor between 0.9 and 1.0;

- adjusting the brightness of the image by $-10 \%$ to $+10 \%$, truncating the brightness of each pixel afterwards between 0 and 1 ;

- translation from the original position by -4 to +4 pixels in the horizontal and vertical directions (the maximum shift is fairly small to avoid pushing the image out of bounds).

The training data are available for replication purposes. The details can be found under Code and data availability at the end of the article. 


\section{Methods}

In this section, we provide a brief overview of the existing techniques we applied in our classification scheme. The specific implementation details and novel methodology used in this study can be found in Sect. 4 .

\subsection{Convolutional neural networks}

The development of the theory and best practices for CNNs has rapidly enhanced the capacity of computers to process spatially structured data such as images (LeCun et al., 2015). These networks employ a series of convolution operations and nonlinearities to extract successively higher-level features of images. Each such operation is called a layer of the network. The most common types of layers are described briefly below:

Dense layers (also called fully connected layers) map their input vector $\boldsymbol{x}$ to the output $\boldsymbol{y}$ as an affine transformation $\boldsymbol{y}=\mathbf{W} \boldsymbol{x}+\boldsymbol{b}$, where the matrix $\mathbf{W}$ and vector $\boldsymbol{b}$ consist of trainable parameters.

Convolution layers map the channels of their input to their output as a sum of convolution operations. The convolution kernels are trainable parameters that are learned by the network.

Activation layers apply a nonlinear function to their input. This, in combination with the mixing operation implemented by the convolution and/or dense layers, allows the network to learn highly nonlinear maps when enough layers are used. The most common activation function in CNNs is the rectified linear unit (ReLU; Nair and Hinton, 2010), which is defined as

$a(x)= \begin{cases}0, & x<0 \\ x, & x \geq 0 .\end{cases}$

In this work, we used a variant called Leaky ReLU:

$a(x)= \begin{cases}\alpha x, & x<0 \\ x, & x \geq 0,\end{cases}$

where the hyperparameter $\alpha$ is a small positive number that is used to permit a small but nonzero gradient at $x<0$.

Pooling layers reduce the spatial dimensions of their input by dividing it into $M \times N$ (typically $2 \times 2$ ) rectangular regions arranged in a grid and then applying a pooling operation such that each rectangle is mapped to a single value in the output image. Usually, either the average or the maximum of the rectangle is used as the pooled value. Pooling operations can sometimes be replaced by strided convolutions, which skip some points (e.g., every other point) of the input to reduce the spatial dimensionality of the output.
Normalization layers seek to normalize their input data, usually trying to constrain the input to optimal mean and variance (which are typically either fixed to 0 and 1 , respectively, or optimized as parameters of the network). This seeks to keep the variables in the active (i.e., nonzero gradient) ranges of the activation functions and to reduce the dependence between the parameters of the network. Common normalization strategies include batch normalization (Ioffe and Szegedy, 2015) and instance normalization (Ulyanov et al., 2017a). More recently, Huang and Belongie (2017) introduced adaptive instance normalization (AdaIN), which allows the network to be adapted to different styles through external weighting of layers.

In practice, layers are often organized in blocks, predefined series of operations implemented using the abovementioned types of layers. Recently, residual network (or "ResNet") blocks - which add their input to their output, allowing the nonlinear part to operate only on the residual - have been popular after it was found that they improve accuracy over similar-sized non-residual networks in classification tasks (He et al., 2016).

Neural networks are trained by minimizing a loss function through gradient descent, where gradients are evaluated using the backpropagation algorithm (Rojas, 1996). For a more comprehensive and technical introduction to CNNs, we refer the reader to, for example, Goodfellow et al. (2016).

\subsection{Generative adversarial networks}

A GAN is a system of two neural networks, usually CNNs, that are trained adversarially, i.e., competing against each other. One network, the discriminator $D$, is a binary classifier that is optimized to distinguish inputs that belong to the training dataset ("real") from those that do not ("fake"). The other network, the generator $G$, is simultaneously trained to produce artificial outputs that the discriminator considers to be real. Thus, the generator learns to produce examples that look real to the discriminator and hence resemble the examples found in the training dataset. A sufficiently large dataset is needed such that neither the discriminator nor the generator can simply memorize the set of input images but must instead learn the structure of the inputs. The input to the generator, the noise $z$, is sampled from a simple probability distribution; we use a multivariate standard normal distribution in this work.

The initially proposed GAN loss (Goodfellow et al., 2014) treats $D$ as a probabilistic binary classifier where the output $D(\boldsymbol{x}) \in[0,1]$, representing the estimated probability of $\boldsymbol{x}$ being a fake rather than a real sample. Using binary crossentropy, the GAN losses can then be written as

$$
\begin{aligned}
L_{\mathrm{D}}\left(\boldsymbol{x}, \boldsymbol{z} ; \boldsymbol{\theta}_{\mathrm{D}}\right) & =\log (D(\boldsymbol{x}))-\log (1-D(G(\boldsymbol{z}))), \\
L_{\mathrm{G}}\left(z ; \boldsymbol{\theta}_{\mathrm{G}}\right) & =\log (1-D(G(\boldsymbol{z}))),
\end{aligned}
$$


where $L_{\mathrm{D}}$ is the discriminator loss and $L_{\mathrm{G}}$ the generator loss, and $\boldsymbol{\theta}_{\mathrm{D}}$ and $\boldsymbol{\theta}_{\mathrm{G}}$ are the corresponding trainable weights. The optimization goals are

$$
\begin{array}{ll}
\min _{\boldsymbol{\theta}_{\mathrm{D}}} & \mathrm{E}_{\boldsymbol{x}, z}\left[L_{\mathrm{D}}\left(\boldsymbol{x}, \boldsymbol{z} ; \boldsymbol{\theta}_{\mathrm{D}}\right)\right], \\
\min _{\boldsymbol{\theta}_{\mathrm{G}}} & \mathrm{E}_{z}\left[L_{\mathrm{G}}\left(z ; \boldsymbol{\theta}_{\mathrm{G}}\right)\right] .
\end{array}
$$

As the goals are mutually contradictory, the training proceeds by alternating between training $D$ with fixed $\boldsymbol{\theta}_{\mathrm{G}}$ and training $\boldsymbol{G}$ with fixed $\boldsymbol{\theta}_{\mathrm{D}}$.

More recently there have been developments that seek to provide a more stable loss for GANs. The Wasserstein GAN (WGAN; Arjovsky et al., 2017) is motivated by the Wasserstein distance between probability distributions, and its losses can be written simply as

$$
\begin{aligned}
L_{\mathrm{D}}\left(\boldsymbol{x}, \boldsymbol{z} ; \boldsymbol{\theta}_{\mathrm{D}}\right) & =D(\boldsymbol{x})-D(G(\boldsymbol{z})), \\
L_{\mathrm{G}}\left(\boldsymbol{z} ; \boldsymbol{\theta}_{\mathrm{G}}\right) & =D(G(\boldsymbol{z})) .
\end{aligned}
$$

Thus with WGAN, the discriminator is trained to make its output as small as possible for real inputs and as large as possible for generated inputs. A slight variant called the "hinge loss" was employed to regularize the discriminator by Donahue and Simonyan (2019) for very high image quality in the context of unsupervised feature learning:

$$
\begin{aligned}
L_{\mathrm{D}}\left(\boldsymbol{x}, \boldsymbol{z} ; \boldsymbol{\theta}_{\mathrm{D}}\right) & =h(-D(\boldsymbol{x}))+h(D(G(z))), \\
h(t) & =\max (0,1-t) .
\end{aligned}
$$

WGANs can be constrained by combining them with gradient penalty, which regulates the gradients of the discriminator outputs, with respect to the inputs, by penalizing them for deviating from unit length (Gulrajani et al., 2017). This yields an additional term for the discriminator loss as

$L_{\mathrm{GP}}\left(\boldsymbol{x}, \boldsymbol{z} ; \boldsymbol{\theta}_{\mathrm{D}}\right)=\left(\left\|\nabla_{\hat{\boldsymbol{x}}} D(\hat{\boldsymbol{x}})\right\|_{2}-1\right)^{2}$.

The samples $\hat{\boldsymbol{x}}$ are randomly weighted averages between real and generated samples:

$\hat{\boldsymbol{x}}=\epsilon \boldsymbol{x}+(1-\epsilon) G(\boldsymbol{z})$,

where $\epsilon$ is a random number sampled from the uniform distribution between 0 and 1 .

\subsection{Latent-variable extraction with GANs}

The generator mapping from the simple probability distribution of $\boldsymbol{z}$ to the complex distribution of $\boldsymbol{x}$ is not invertible in the basic GAN formulation. However, mapping $x$ to $z$ (or a subset of $z$, which we denote as $z_{1}$ ) is of great interest to unsupervised learning as it allows the essential features of the training images to be encoded into much simpler vectors in the latent distribution. Consequently, several GAN variants have been proposed that incorporate an approximate inverse mapping from $x$ to $z_{1}$ using various approaches (e.g., Chen et al., 2016; Donahue et al., 2017; Ulyanov et al., 2017b).

\subsection{Classification: $K$-medoids}

$K$-medoids (Jain et al., 1999), similar to the more commonly used $K$-means (Kaufman and Rousseeuw, 1990), is an unsupervised classification method that seeks to associate each point $\boldsymbol{y}_{i}$ in a dataset with one of $K$ center points. Thus, data points that are close to each other tend to be associated with the same center point, and thus they can be considered to be members of the same class.

The $K$-medoids and $K$-means algorithms both select the center points $\boldsymbol{c}_{j}, j=1 \ldots K$, to minimize the cost

$$
\begin{aligned}
L & =\frac{1}{N} \sum_{i=1}^{N} d\left(\boldsymbol{y}_{i}, \boldsymbol{c}_{\mathrm{n}, i}\right), \\
\boldsymbol{c}_{\mathrm{n}, i} & =\underset{\boldsymbol{c}_{j}}{\operatorname{argmin}} d\left(\boldsymbol{y}_{i}, \boldsymbol{c}_{j}\right),
\end{aligned}
$$

where $d$ is a distance metric between two points and $\boldsymbol{c}_{\mathrm{n}, i}$ denotes the center point that is nearest to data point $\boldsymbol{y}_{i}$. In other words, the center points are chosen such that the average distance of each point $\boldsymbol{y}_{i}$ to $\boldsymbol{c}_{\mathrm{n}, i}$ is minimized.

The standard $K$-means algorithm uses the squared $\mathrm{Eu}$ clidean distance (SED) metric

$d_{K \text {-means }}\left(\boldsymbol{y}_{1}, \boldsymbol{y}_{2}\right)=\left|\boldsymbol{y}_{1}-\boldsymbol{y}_{2}\right|^{2}$.

It is called $K$-means because it can be seen as a generalization of the $K=1$ case where the unique optimal center point is simply the mean of the data points. In contrast to that special case, with $K \geq 2$ the solution is less straightforward and must be found iteratively.

The $K$-medoids algorithm can be understood as a variant of $K$-means. Unlike with $K$-means, where a center point can be an arbitrary point in space, the $K$-medoids algorithm selects $K$ data points to act as centers ("medoids"). The advantage of this is that an arbitrary distance metric $d$ between points can then be used. The partitioning-around-medoids algorithm (PAM; Kaufman and Rousseeuw, 1990) starts with randomly selected medoids and finds the optimum by iterating the following steps:

1. For each medoid $\boldsymbol{c}_{j}$,

a. compute $L_{i j}$ as the values that $L$ would have if $\boldsymbol{y}_{i}$ were used as $\boldsymbol{c}_{j}$ instead;

b. if the cost improves, i.e., $\min _{i} L_{i j}<L$, set $\boldsymbol{c}_{j}:=$ $\boldsymbol{y}_{i, \mathrm{opt}}$, where $i_{\mathrm{opt}}=\operatorname{argmin}_{i} L_{i j}$.

2. If $L$ decreased in step (1), repeat it. Otherwise, terminate.

This iteration, like that of $K$-means, is not guaranteed to find the globally optimal solution; restarting the algorithm multiple times and selecting the best solution (smallest $L$ ) is often helpful. Furthermore, the number of center points must be set manually; it is not inferred by the algorithm. 


\section{Implementation}

\subsection{GAN architecture and training}

Following Donahue and Simonyan (2019), we implemented the GAN using the hinge loss (Eq. 10) and gradient penalty (Eq. 12) for the discriminator and the WGAN loss (Eq. 7) for the generator. We also added a second output $D_{\mathrm{E}}$ to the discriminator to extract the latent variables received by the generator. To enforce the recovery of the latent variables, we added a mean-square-error (MSE) loss between the original and recovered latent variables to both $D$ and $G$, similar to InfoGAN:

$L_{\mathrm{MSE}}\left(\boldsymbol{x}, \boldsymbol{z} ; \boldsymbol{\theta}_{\mathrm{D}}, \boldsymbol{\theta}_{\mathrm{G}}\right)=\left|D_{\mathrm{E}}(G(z))-z_{1}\right|^{2}$,

where $z_{1}$ is the subset of $z$ that the discriminator attempts to recover. The complete GAN losses are then

$$
\begin{aligned}
L_{\mathrm{D}}\left(\boldsymbol{x}, \boldsymbol{z} ; \boldsymbol{\theta}_{\mathrm{D}}\right) & =h(-D(\boldsymbol{x}))+h(D(G(\boldsymbol{z}))) \\
& +\gamma L_{\mathrm{GP}}\left(\boldsymbol{x}, \boldsymbol{z} ; \boldsymbol{\theta}_{\mathrm{D}}\right) \\
& +\beta L_{\mathrm{MSE}}\left(\boldsymbol{x}, \boldsymbol{z} ; \boldsymbol{\theta}_{\mathrm{D}}, \boldsymbol{\theta}_{\mathrm{G}}\right), \\
L_{\mathrm{G}}\left(\boldsymbol{x}, \boldsymbol{z} ; \boldsymbol{\theta}_{\mathrm{G}}\right) & =D(G(\boldsymbol{z}))+\beta L_{\mathrm{MSE}}\left(\boldsymbol{x}, \boldsymbol{z} ; \boldsymbol{\theta}_{\mathrm{D}}, \boldsymbol{\theta}_{\mathrm{G}}\right),
\end{aligned}
$$

where $\gamma$ is the weight of the gradient penalty and $\beta$ is the weight of the MSE loss. We used $\gamma=10$ following Gulrajani et al. (2017) and $\beta=1$ as we found that this simple choice led to good convergence of both the GAN image generation and the latent-variable recovery.

The discriminator (Fig. 1a) is a series of six ResNettype blocks of layers, each consisting of two activationconvolution blocks. Those blocks transform the image to 512 feature maps of $4 \times 4$ size. The convolutional blocks are followed by a pooling layer and two fully connected layers. From these, the discriminator output $D$ and the latentencoder output $D_{\mathrm{E}}$ are derived. Spectral normalization (Miyato et al., 2018) is used in all layers of the discriminator. The batch-statistics technique suggested by Karras et al. (2019) is also used to encourage variety and inhibit mode collapse, a failure where the generator always generates the same (or one of a few) outputs.

The generator (Fig. 1b) derives from the StyleGAN concept of Karras et al. (2019). The input to the generator, $z$, is made up of two components: the vector of latent variables $z_{1}$ that is recovered by the discriminator and an additive noise component $z_{\mathrm{a}}$ that is not recovered. The generator begins the processing chain by transforming the latent vector $z_{1}$ into initial feature maps of $4 \times 4$ size. This is then processed through five blocks of layers, each of which contains the following operations:

- Upsampling layers increase the pixel size of the feature maps by a factor of 2 each. After the total of five upsampling operations the size is increased by a factor of $2^{5}=32$, thus transforming the initial $4 \times 4$ feature maps to $128 \times 128$ size.
- Convolution and activation layers transform the input into successively lower levels of representation; collectively these perform the transformation from the feature representation to the pixel representation.

- AdaIN layers reweight the features such that, at each level of processing, the appropriate features for the given snowflake type are selected; this is referred to as "styling". After the styling, the additive noise $z_{\mathrm{a}}$ is applied. The objective of this is to capture the essential features of the snowflake in the style vector, while the less significant variation between individual snowflakes of the same type is represented in the noise. However, contrary to Karras et al. (2019), we found the additive noise to have an insignificant effect on the final generated images. Also deviating from Karras et al. (2019), the styling blocks are residual, adding the result to the input at the end of processing, as we found that this improved convergence.

We trained the GAN by alternating between training the discriminator with a single batch of data with the generator weights held constant and training the generator with a single batch with constant discriminator weights. We used a batch size of 64 for both networks. The training was monitored manually and terminated when neither the losses nor the image quality was any longer changing appreciably. The transformations and augmentations described in Sect. 2 were applied to each batch before processing. We used the Adam optimizer (Kingma and Ba, 2015) with the learning rate set to $10^{-4}$ for both the discriminator and the generator. We trained the network with Nvidia Tesla P100 and K40 graphics processing units (GPUs); the final training used to derive the results presented in this article took approximately $72 \mathrm{~h}$ on the $\mathrm{K} 40$, while training on the $\mathrm{P} 100$ was roughly twice as fast.

\subsection{Clustering}

In principle, we could have used $K$-means classification to derive classes directly from the extracted latent variables $z_{1}$. However, we found that the latent variables code for some properties of the images that one should not actually use for classification: for example, some of the variation in $z_{1}$ corresponds to the orientation of the particle, while a given particle should belong to the same class regardless of the orientation at which it is seen. Therefore, the variation in $z_{1}$ corresponding to these properties should be excluded from the classification.

We found that the unsupervised classification can be made more robust and approximately invariant to unwanted image properties by producing random variations of each image and then associating each image with a distribution of points rather than a single point in the latent space. Specifically, in our classification scheme the random augmentations described in Sect. 2 (none of which should affect the classification) are applied to each image 100 times, extracting the 
(a) Discriminator

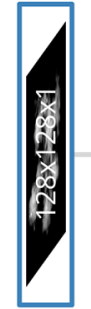

Image

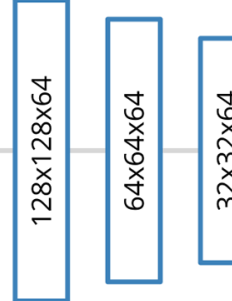

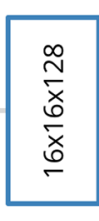
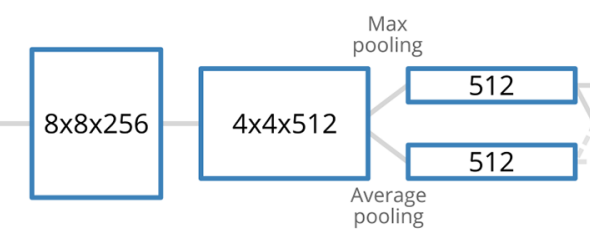

西

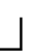

Dense blocks

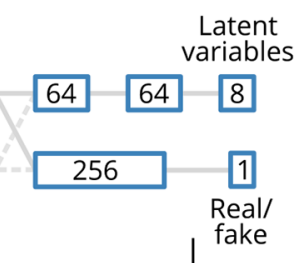

fake

Residual convolution blocks

(b) Generator

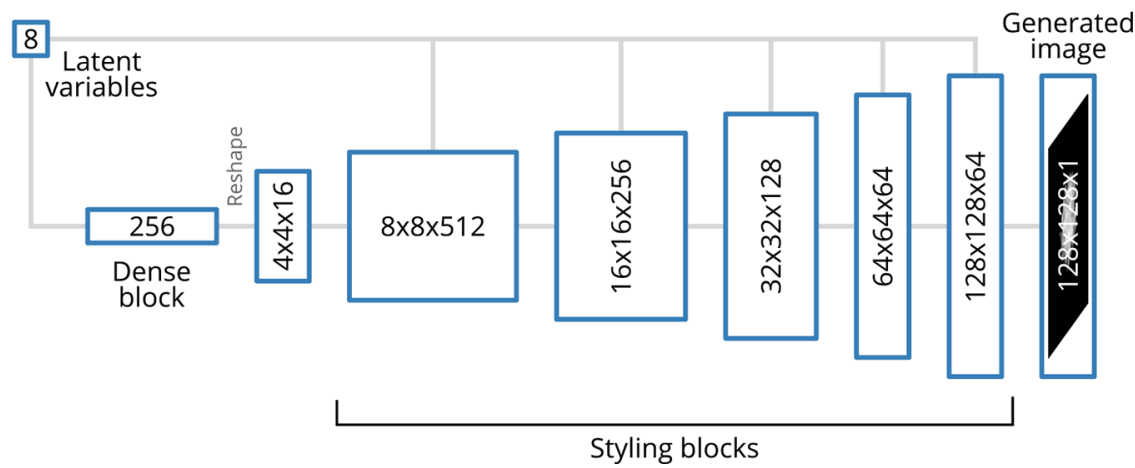

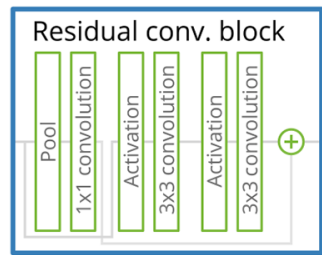

Dense block

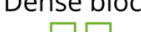

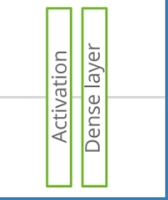

Figure 1. Outlines of the architectures of the (a) discriminator and (b) generator networks. The numbers on the blocks denote the size and number of feature maps; for example, $16 \times 16 \times 256$ indicates 256 feature maps of $16 \times 16$ pixels each. The number of features for each layer was determined by adapting network architectures used in earlier GAN studies and then tuning the sizes to achieve a reasonable compromise between performance and computational cost.

latent variables $z_{1}$ for each variation. From these samples, the mean $\boldsymbol{\mu}_{i}$ and covariance $\boldsymbol{\Sigma}_{i}$ of the latent variables for each image $i$ are computed. Now, an approximately augmentationinvariant distance can be defined between two snowflake images using a metric for the distance between two probability distributions. For this, the Bhattacharyya distance between two multivariate normal distributions (Fukunaga, 1990) is adopted:

$$
\begin{gathered}
d_{\mathrm{B}}\left(\boldsymbol{x}_{i}, \boldsymbol{x}_{j}\right)=\frac{1}{8}\left(\boldsymbol{\mu}_{i}-\boldsymbol{\mu}_{j}\right)^{T} \boldsymbol{\Sigma}^{-1}\left(\boldsymbol{\mu}_{i}-\boldsymbol{\mu}_{j}\right) \\
+\frac{1}{2} \ln \left(\frac{\operatorname{det} \boldsymbol{\Sigma}}{\sqrt{\operatorname{det} \boldsymbol{\Sigma}_{i} \operatorname{det} \boldsymbol{\Sigma}_{j}}}\right), \\
\boldsymbol{\Sigma}=\frac{\boldsymbol{\Sigma}_{i}+\boldsymbol{\Sigma}_{j}}{2} .
\end{gathered}
$$

While the distribution of latent-space points for each particle is not guaranteed to be normal, we adopted this distance metric because it is reasonably fast to compute and symmetric with respect to a swap of $i$ and $j$. It is also a generalization of the SED (Eq. 16) in the following sense: if $\boldsymbol{\Sigma}_{i}=\boldsymbol{\Sigma}_{j}=a \mathbf{I}$ for any $a>0, d_{\mathrm{B}}$ is linearly proportional to the SED between the means, $\left|\boldsymbol{\mu}_{i}-\boldsymbol{\mu}_{j}\right|^{2}$.
Additionally, we adopted a simple hierarchical clustering algorithm to iteratively reduce the number of classes derived from the $K$-medoids algorithm by forming a binary tree structure. This algorithm proceeds bottom-up as follows:

1. Start with $K$ separate branches, each containing one of the medoids.

2. On each iteration,

(a) find the pair of branches $(j, k)$ with the shortest distance $d(j, k)$ between the two branches;

(b) record $j$ and $k$, and then merge branch $k$ into branch $j$.

The iteration can be continued until all branches have been merged into a single tree structure. The distance between two branches, $d(j, k)$, can be defined in multiple ways, but we found that the best results were obtained by defining it as the average of all possible $d_{\mathrm{B}}$ between medoids belonging to branch $j$ and those belonging to branch $k$.

After classification, the medoids are reordered manually such that the tree structure resulting from the hierarchical clustering can be visualized clearly. This change is purely cosmetic and does not affect any metrics. 

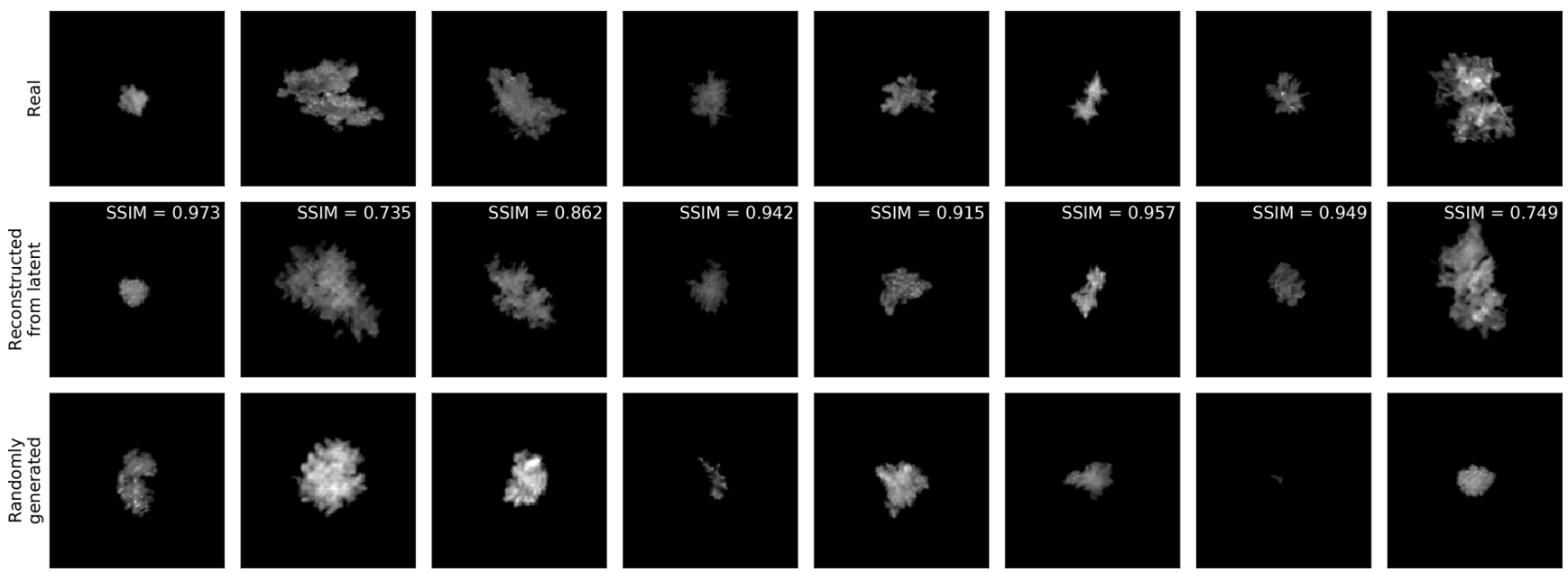

Figure 2. Samples of real and generated images. Top row: real images from the dataset. Middle row: images generated by extracting the latent variables from the corresponding top-row image and then generating an image with the GAN generator from that image; the SSIM between the real and generated images is also shown. Bottom row: samples of snowflake images generated from random latent codes.

\section{Results and discussion}

\subsection{Image generation from latent variables}

Figure 2 shows samples of real and generated images. The middle row of Fig. 2 displays a reconstruction of the real snowflake shown on the top row. The reconstructed image is obtained by extracting the latent variables from the original image with $D_{\mathrm{E}}$ and then generating an image from the latent variables using $G$. The reconstruction is not perfect - nor can that be expected given that $D_{\mathrm{E}}$ compresses each $128 \times 128$ pixel image to only eight real numbers - but the reconstructed images are quite similar to the corresponding originals, showing that the latent codes encapsulate information about the essential features of the image such as size, shape, contrast, orientation and texture. The bottom row of Fig. 2 shows snowflakes generated using randomly selected latent variables. These images also look qualitatively plausible, demonstrating that the generator is not dependent on the latent variables being extracted from a real image.

The generator does not replicate all features found in the original snowflake. For instance, fine details of the large aggregate in the rightmost column of Fig. 2 are not reproduced in the reconstructed image. This is consistent with the relatively low structural similarity index (SSIM; Wang et al., 2004) of 0.749 between these two images. The average structural similarity index between real and generated images in the dataset is 0.928 . However, in contrast to most applications of GANs, in this study the image generation is merely a byproduct of the classification scheme. The primary goal is to train the discriminator to extract latent variables that can be used for classification.

The changes to the generated image caused by varying the latent variables are shown in Fig. 3. Two latent variables are varied while the rest are held constant. This gives an example of how the GAN maps the latent variables to the data distribution. The generated images look plausible at each combination of the two latent variables, while the image changes smoothly between two different shapes of aggregate (top left and right corners), a large rimed column-like snowflake (bottom left corner) and a small irregular snowflake (bottom right corner). This ability of the GAN to learn an encoding between the latent variables and the essential image properties is the basis of the classification.

\subsection{Classification}

\subsubsection{The number of classes}

A common problem with unsupervised classification is selecting the number of classes. Using the latent variables extracted by the GAN, we ran the $K$-medoids classification, as described in Sect. 4.2, for values of $K$ between 1 and 20 and recorded the change in the cost function $L$ (Eq. 15). Since the computational complexity of the $K$-medoids algorithm scales as $\mathcal{O}\left(N^{2}\right)$, and therefore it would be very expensive to run it for the entire dataset, we subsampled 2048 random images from the dataset (the same subset was used for each $K$, but similar classification results were obtained with different subsets). The best solution for each $K$ was found by running the algorithm until convergence and then restarting it repeatedly until eight restarts were performed without $L$ decreasing. The solution with the smallest $L$ was selected, and the others discarded.

The loss, as a function of $K$, is shown as the blue solid line of Fig. 4. With clustered data, such analysis often reveals the appropriate number of medoids, as $L$ decreases sharply until the number of medoids reaches the number of clusters and much more slowly afterwards. In Fig. 4, no such threshold 


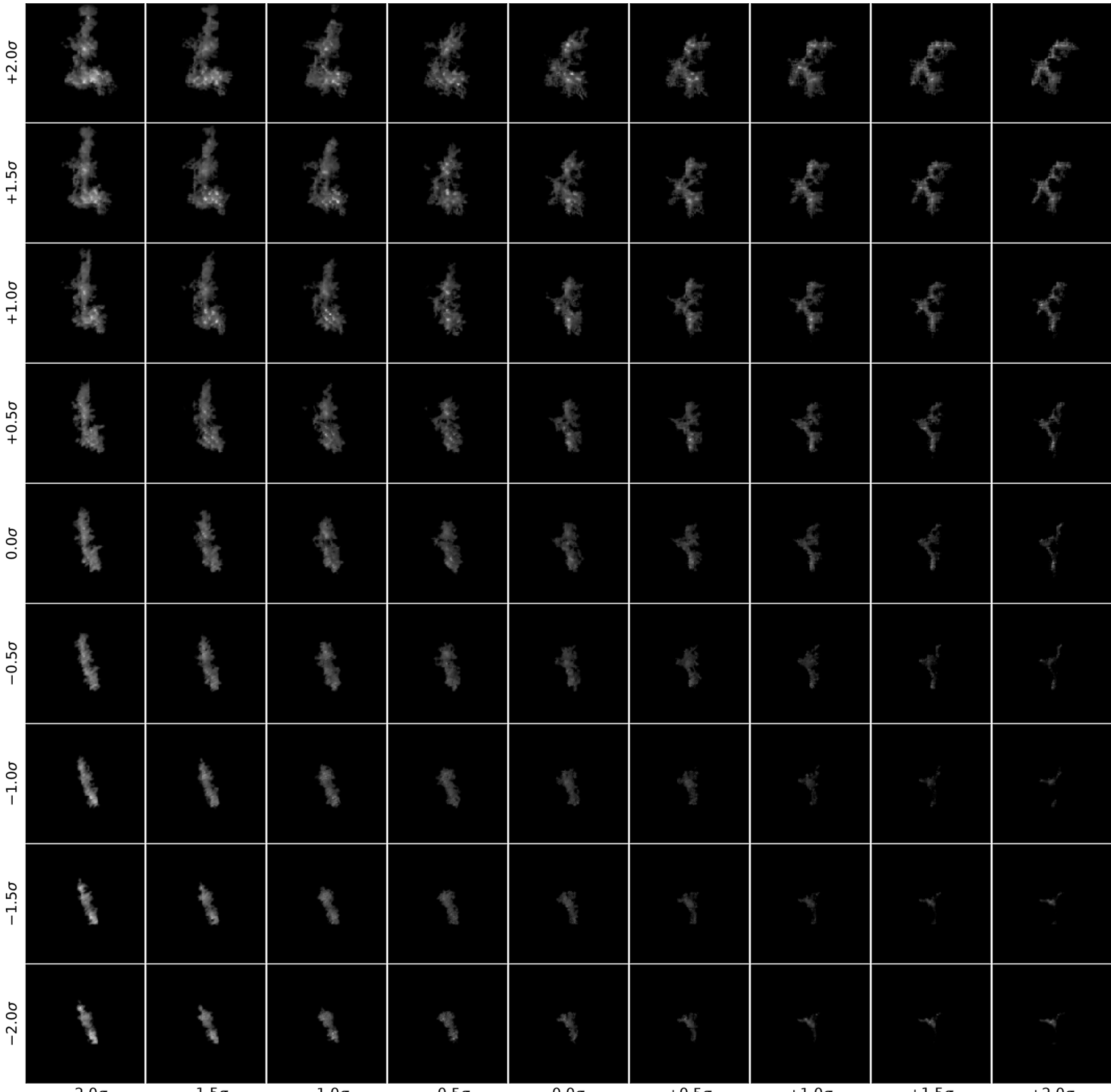

$-2.0 \sigma$

$-1.5 \sigma$

$-1.0 \sigma$

$-0.5 \sigma$

$0.0 \sigma$

$+0.5 \sigma$

$+1.0 \sigma$

$+1.5 \sigma$

$+2.00$

Figure 3. An example of the effect of varying the latent variables. Two latent variables of the GAN are varied from -2 to +2 standard deviations $(\sigma)$, while the others are held constant.

is apparent. Instead, the loss decreases gradually and monotonically as $K$ increases, with diminishing returns at higher $K$.

Given that examining $L$ as a function of $K$ does not suggest any obvious choice for the number of medoids, we needed to select it subjectively. However, we could start with a relatively large $K$ and iteratively simplify the classification using hierarchical clustering, merging nearby classes. The orange dashed line and the green dotted line of Fig. 4 show the results of this procedure on the cost function (starting from $K=16$ and $K=6$, respectively). For the purpose of calculating the cost, a new medoid was determined for each merged class such that it minimizes the sum of distances $d_{\mathrm{B}}$ from the medoid to the members of the newly merged class. 


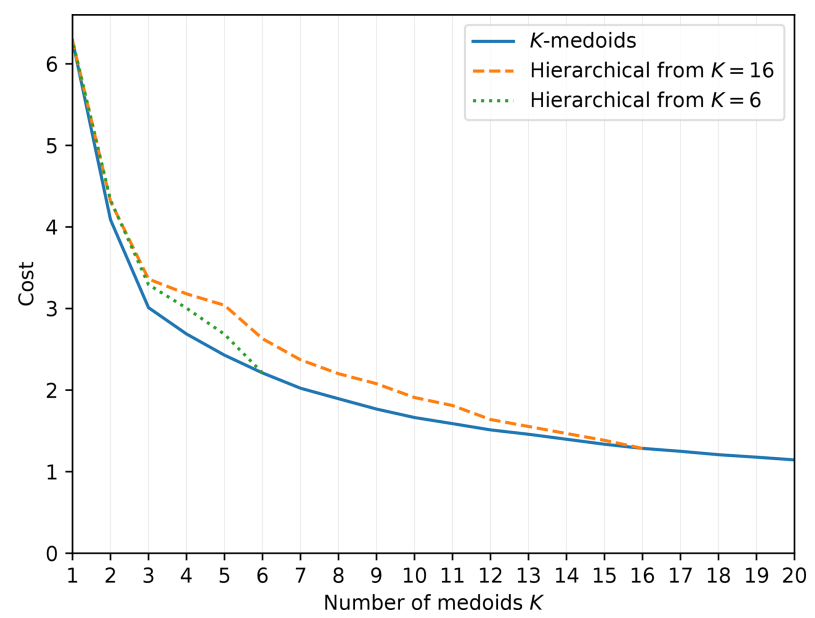

Figure 4. The behavior of the $K$-medoids loss $L$ as a function of the number of medoids $K$. Blue solid line: the results of the $K$-medoids algorithm for each $K$. Orange dashed line: the result of starting with the $K=16$ result and applying hierarchical clustering (as described in Sect. 4.2). Green dotted line: as above but starting with $K=6$.

\subsubsection{Characteristics of classes}

Since there did not appear to be clear reasons to prefer any of the large values of $K$ over the others, we chose $K=16$ somewhat arbitrarily as a value that gives good intra-class similarity of the snowflakes while keeping the classes distinguishable from each other. Samples of class members from the 16-class classification, along with the class hierarchy tree derived using hierarchical clustering, are shown in Fig. 5. The class distance matrix, showing the Bhattacharyya distance between the class medoids, is shown in Fig. 6. Each class contains snowflakes with similar size, shape and texture.

The hierarchical clustering groups the classes into three main branches (classes 1-5, 6-12 and 13-16). The systematic difference between these branches is in the size of the snowflakes, while the variability within each branch reveals differences in the structure of the snowflakes.

The first branch (classes 1-5) consists mostly of large and medium-sized aggregates with some large single crystals. Classes 1,2 and 5 are composed mainly of moderately rimed aggregates, with the main differences among these being size and complexity, both decreasing from class 1 to 2 and further from 2 to 5 . Snowflakes in class 3 are highly complex but less rimed than those of class 1 , while those in class 4 are the most heavily rimed in the first branch. The distances among these classes are all relatively short, and they stand out in the top left corner of Fig. 6.

The second branch (classes 6-12) contains various ice particles smaller than those in the first branch. Classes 6 and 7 contain mostly heavily rimed snowflakes, including graupel, while classes 8-10 are made up of small aggregates and irregular snowflakes, with those in classes 8 and 9 being of similar size and those in class 10 somewhat smaller. The distances among these classes are very short. Class 11 resembles the classes of the first branch (as evidenced by its short distance to classes 3 and 5), containing medium-sized aggregates with little or no riming. Finally, class 12 is similar to class 11 but with slightly smaller snowflakes.

The smallest particles are found in the third branch (classes 13-16). Classes 13 and 15 contain small rimed crystals and graupel, while class 14 differs from those by being unrimed or lightly rimed. Class 16 is the most visually distinct of all classes and is composed mainly of columnar crystals.

Figure 7 shows the memberships in each class. Fewer snowflakes are classified into the more extreme classes consisting of either very large or very small snowflakes. The APRES3 data contain larger proportions of the smallsnowflake classes. There are also more rimed snowflakes in the APRES3 data, consistent with the observations of Del Guasta et al. (1993) and Grazioli et al. (2017) that mixed-phase clouds and riming were frequent in Dumont D'Urville.

In Fig. 8, we show the same type of classification as Fig. 5, but performed with only 6 classes in order to see how the classes combine. While the columnar crystals are again well separated from other types, there is considerably more variability within each class. Particularly importantly, various degrees of riming become more mixed within the classes. Therefore, if one wants to derive information about the microphysics, especially riming, it appears to be preferable to start with a relatively large number of classes and merge them later either in an automated fashion (e.g., the hierarchical clustering shown here) or more subjectively (see Sect. 5.2.3 below). Accordingly, we will concentrate on the 16-class scheme in the rest of this article. The other classifications can be found in the released data.

\subsubsection{Microphysical classification}

As mentioned above, it seems preferable to perform the classification with a fairly large number of classes and then merge them as needed. While this can be done in an objective fashion using the hierarchical clustering, the algorithms perform the analysis based only on the image properties and have no knowledge of the underlying microphysical processes. Therefore, subjective categorization of the classes based on expert analysis can also be helpful. Although this adds a manual component to the classification process, it greatly reduces the amount of work required compared to a supervised approach because the expert only needs to interpret a small number (for us, 16) of classes created by the unsupervised algorithm, rather than having to label a large number (here, hundreds of thousands) of training samples for a supervised algorithm. 


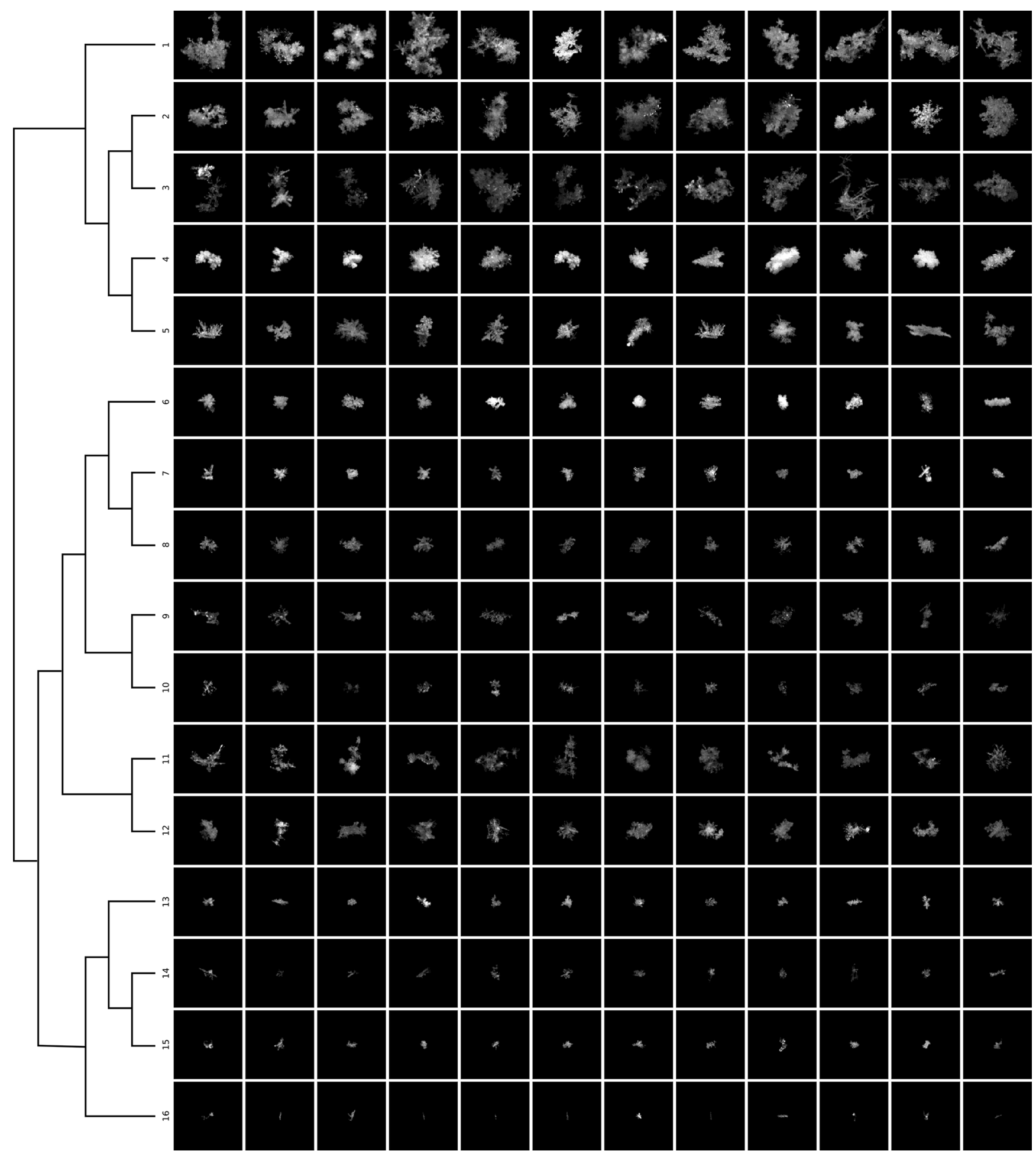

Figure 5. Samples from each class using the 16-class classification. Each row corresponds to a class; the first column shows the particle used as the medoid, while the other columns show random samples. The lines on the left illustrate the tree structure derived with hierarchical clustering. 


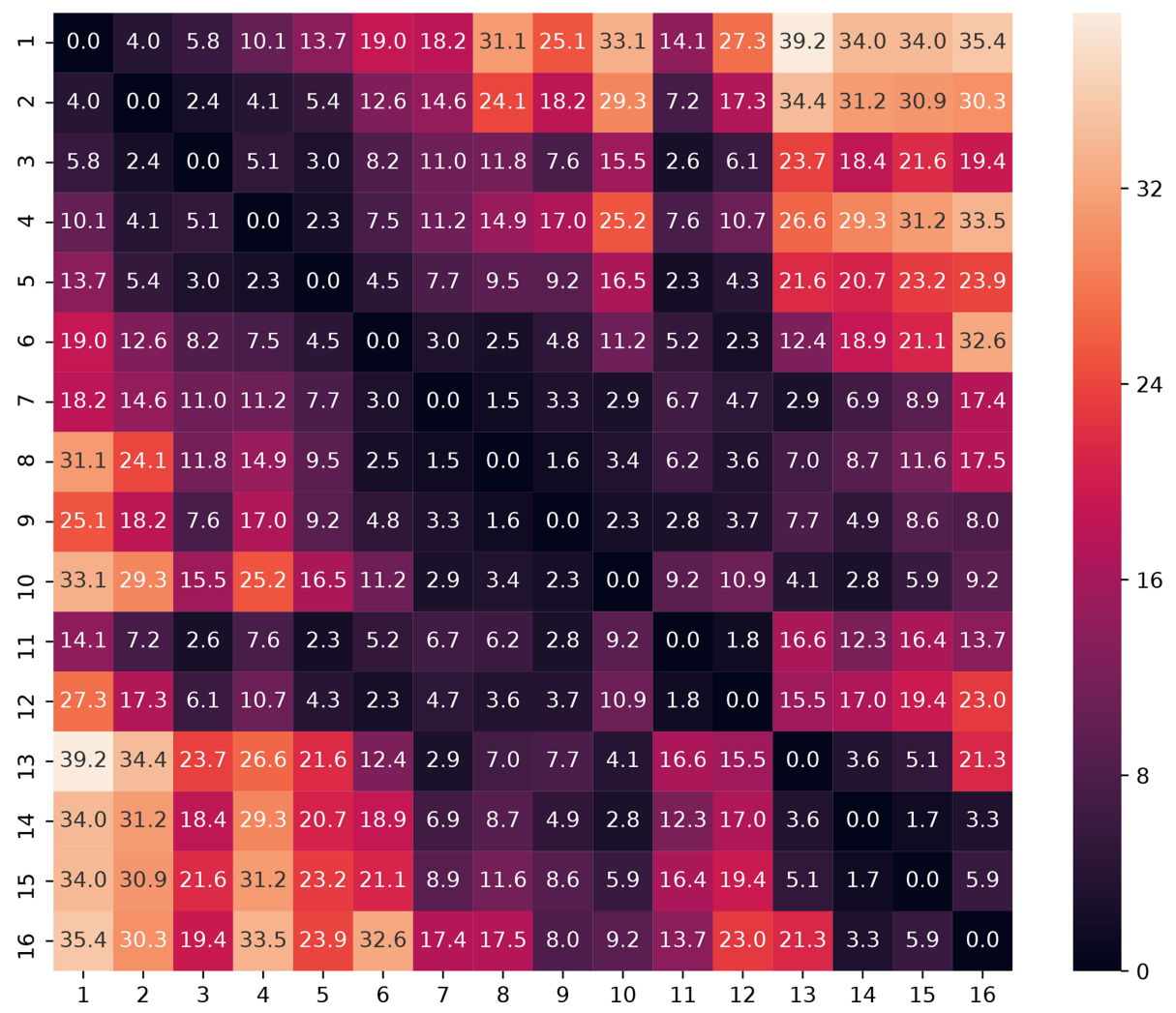

Figure 6. Class distance matrix for the 16-class classification, showing the Bhattacharyya distance between class medoids as a heatmap.

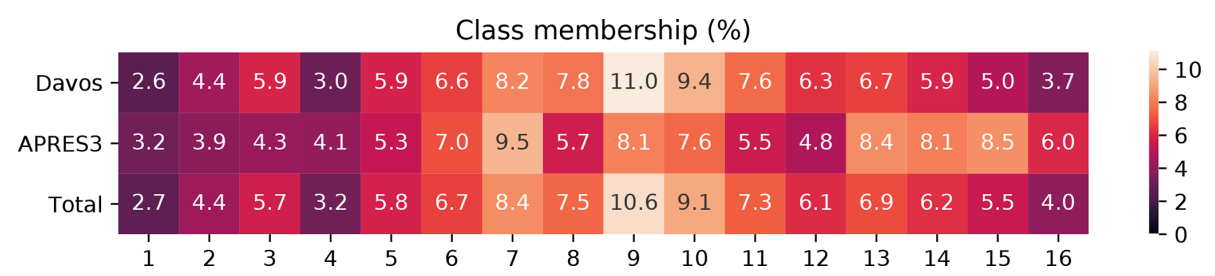

Figure 7. A heatmap showing the percentage of class memberships in each class for the Davos and APRES3 data as well as the entire dataset.

Different applications may require different definitions, but for characterizing snowfall events we suggest the following categories:

1. lightly rimed aggregates: classes 3,8,9,10 and 11;

2. moderately rimed aggregates: classes 1, 2, 5 and 12;

3. large, heavily rimed snowflakes: classes 4, 6 and 7;

4. small, heavily rimed snowflakes: classes 13 and 15;

5. small crystals and their aggregates: class 14;

6. columnar crystals: class 16.

\subsubsection{Comparison to supervised classification}

In order to compare our results to those of the P17 supervised classification algorithm, Fig. 9 shows the corresponding P17 classes for each of our 16 classes, normalized such that the membership counts sum to $100 \%$ for each of our classes. The P17 categorization is generally consistent with the analysis of the microphysical properties that we have presented above, showing that the two different schemes are sensitive to many of the same features in the images. The classes designated as aggregates (categories 1 and 2 in Sect. 5.2.3) are also dominated by aggregates in Fig. 9. The heavily rimed classes (categories 3 and 4) contain, as expected, more graupel than the other classes, although in all of these except for class 15 the aggregates are actually the most common type. This is because aggregates are overrepresented in the dataset as a whole, and also because the P17 scheme draws a distinction between heavily rimed aggregates and graupel, which may be difficult to distinguish in practice. Class 14 is a fairly generic grouping of different types of small particles and accordingly contains a wide mix of unrimed hydrometeor types 


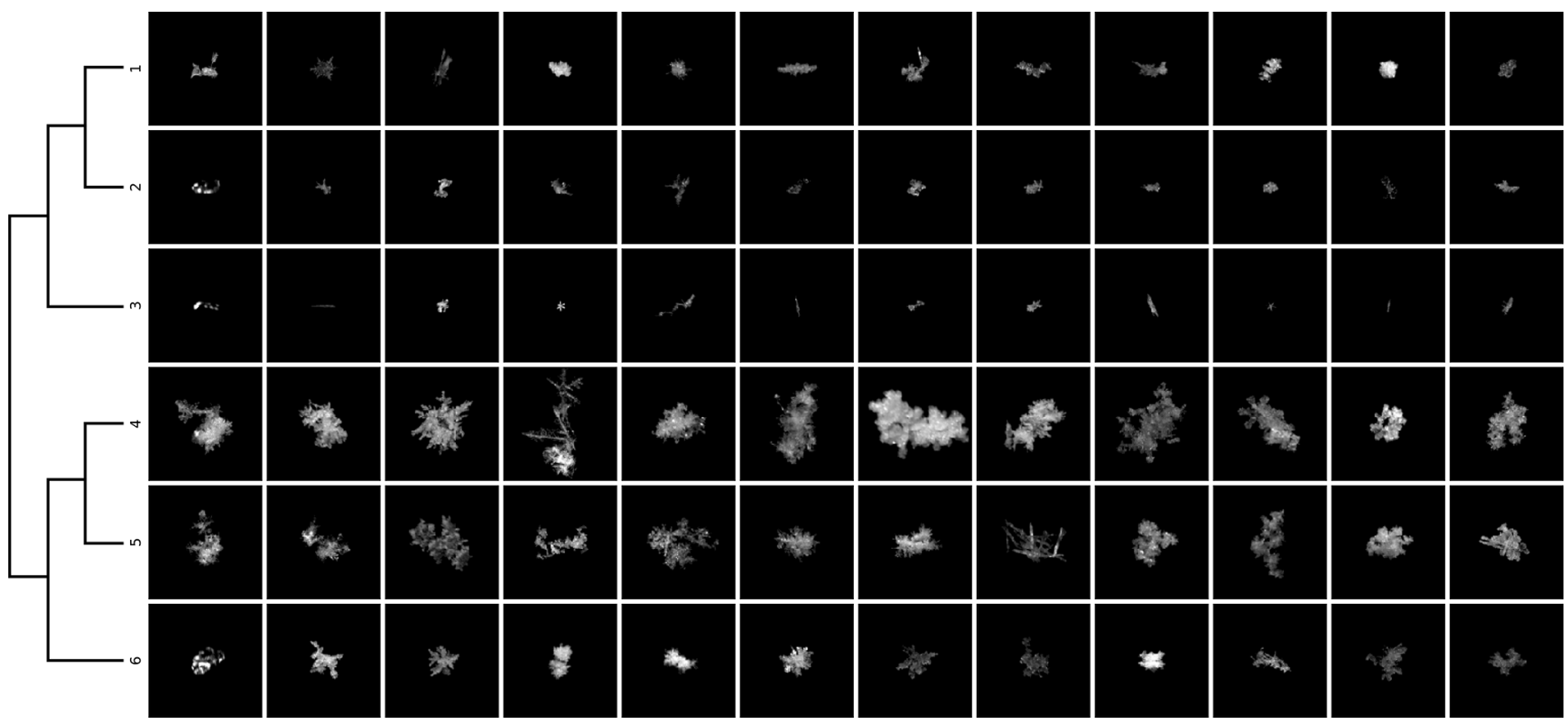

Figure 8. As in Fig. 5 but with $K=6$.

also in the P17 classification. Lastly, class 16 consists mostly of columnar crystals, of which there are very few in the other classes.

\subsubsection{Effectiveness of distribution-based clustering}

The MASC instrument captures images of falling snowflakes using multiple cameras simultaneously. We did not make use of this capability while training the GAN and instead operated with single images, because there is often only one sharp image of a given snowflake and requiring multiple high-quality images of each snowflake would have severely limited the size of our training dataset. However, we could use this capability to evaluate the classification scheme because, ideally, the same snowflake viewed from different angles should result in the same classification.

For each snowflake with multiple angles available, we computed the Bhattacharyya distance $d_{\mathrm{B}}$ between the latentspace distributions (obtained as described in Sect. 4.2) of a pair of images of the same snowflake and the mean of the Bhattacharyya distances to all snowflakes in the dataset. For comparison, we computed the same values for the SED (Eq. 16) between the latent codes obtained without augmentation. Between two images from different angles, median $d_{\mathrm{B}}$ was 1.00 , while median $d_{\mathrm{B}}$ between two different snowflakes was 18.2 (a ratio of 0.055 ). The corresponding median values for the SED were 14.3 for matched pairs and 20.2 for all snowflakes (ratio of 0.71).

Another way to compare the two distance metrics is through the distance rank of a pair of images of the same snowflake, which is defined here as the percentage of all snowflakes whose distance from the given snowflake is longer than the distance between the pair. The median distance rank is $98.5 \%$ for $d_{\mathrm{B}}$ and $70.3 \%$ for SED.

Clearly, using the data augmentation and the distribution distance metric in the latent space brings images of the same snowflake much closer to each other. Consequently, we can expect that this approach significantly increases the reliability of unsupervised classification using the latent variables.

\section{Summary}

MASC instruments have been deployed in diverse locations around the world over the last decade, resulting in datasets comprising millions of high-resolution images of falling snowflakes. Automated analysis is needed to explore such large quantities of data, and advanced image-processing techniques are beneficial because the image structure contains a signature of the microphysical processes that led to the formation of each snowflake. In this work, we have described an unsupervised approach to this problem where a combination of a GAN and $K$-medoids classification is used. The trained GAN is used to map each image into a vector of latent variables that capture the essential properties of the image. The $K$-medoids algorithm is then used to classify the images based on the latent variables, and the number of classes can be reduced to the desired granularity using hierarchical clustering. The GAN also learns to generate artificial images of snowflakes, which we could use to verify that the latent variables map to the properties of snowflakes in a meaningful way.

The latent variables code also for information about the images that one generally does not want to use for classi- 


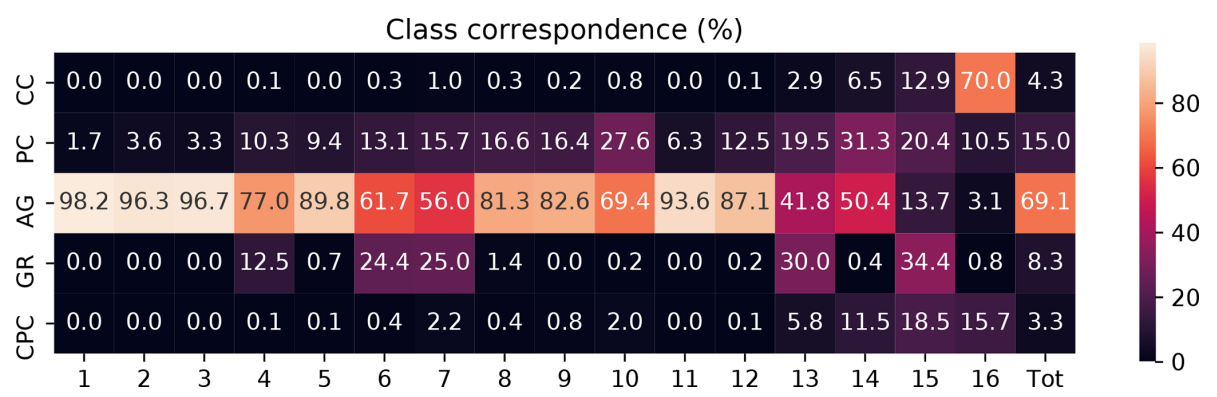

Figure 9. A heatmap showing the correspondence between our 16-class classification and the P17 classes. Each column has been normalized to sum to $100 \%$, and the last column shows the class memberships in the entire dataset. The P17 classes are abbreviated as follows: CC (columnar crystals), PC (planar crystals), AG (aggregates), GR (graupel), and CPC (combinations of plates and columns).

fication, such as the orientation. We mitigated this problem by associating each image with a distribution of latent-space points using data augmentation and defining the distance between images using a distribution distance metric, the Bhattacharyya distance. Using the multiple cameras provided by the MASC, we verified that this results in improved distance estimates between images and consequently more accurate classification.

A qualitative assessment of the classification results confirmed that each class designated by the classification scheme contains snowflakes with microphysical and structural properties similar to other members of the class. Aggregate snowflakes, which make up the majority of the dataset, are divided up into several classes, and the differences between these classes reflect the size and the degree of riming of the aggregates. Columnar crystals and small graupel are also quite well separated from other types of ice particles. The hierarchical clustering results in three main branches that differ from each other mostly in the size of the snowflakes.

Each of the applied methods is unsupervised and consequently can be used without providing labeled training data. Hence, the main advantage of the methodology over supervised classification is that the process can be repeated for new datasets with modest manual effort, albeit at a fairly high computational cost. The unsupervised approach also reduces the role of human experts on the classification. This can have both positive and negative effects, as the effect of human biases is reduced, but simultaneously the potential benefits of expert domain knowledge, i.e., understanding of ice microphysics, are neglected. In practice, we find that our classification approach can help distinguish snowflakes by their microphysical properties, but subsequent analysis is needed to interpret the contents of each class in a microphysical context. Thus, the responsibilities of the domain expert are shifted from creating the training datasets to the less onerous task of interpreting the classification results. The number of classes must also be selected manually, and the class boundaries are somewhat arbitrary as the latent data are not strongly clustered. Therefore, in the future it may be interesting to inves- tigate more continuous classification schemes rather than the discrete classification we have described here.

While our approach to unsupervised classification is based on well-documented machine learning techniques and algorithms, we believe that the combination of methods used here - particularly the use of data augmentation to improve the accuracy of classification using GAN-derived latent variables has not been employed in previous work. We expect that the same methodology can be adapted to the unsupervised classification of many other datasets in different domains.

Code and data availability. The code and the data supporting this project are available at https://github.com/jleinonen/ snow-gan-classification (last access: 26 May 2020, Leinonen, 2019). This repository includes the training datasets, pre-trained models, derived latent variables and code sufficient to replicate the results.

Author contributions. JL and AB formulated the project and developed the methodology used in this study. JL wrote the software code needed to implement and evaluate the method. JL wrote the article with contributions from $\mathrm{AB}$.

Competing interests. The authors declare that they have no conflict of interest.

Acknowledgements. We thank Yves-Alain Roulet and Jacques Grandjean of MeteoSwiss for the MASC data from Davos, and Christophe Praz for assistance with processing the data. We also thank two anonymous reviewers for their constructive comments.

Financial support. This research has been supported by the Swiss National Science Foundation (grant no. 200020_175700). The computational resources used in this work were supported by a grant from the Swiss National Supercomputing Centre (CSCS) under project ID s942. 
Review statement. This paper was edited by Daqing Yang and reviewed by two anonymous referees.

\section{References}

Arjovsky, M., Chintala, S., and Bottou, L.: Wasserstein GAN, arXiv [preprint], arXiv1701.07875, 6 December 2017.

Chen, X., Duan, Y., Houthooft, R., Schulman, J., Sutskever, I., and Abbeel, P.: InfoGAN: Interpretable Representation Learning by Information Maximizing Generative Adversarial Nets, in: Advances in Neural Information Processing Systems 29, edited by: Lee, D. D., Sugiyama, M., Luxburg, U. V., Guyon, I., and Garnett, R., Curran Associates, Inc., 2172-2180, available at: https://papers.nips.cc/paper/6399-infogan-interpretablerepresentation-learning-by-information-maximizing-generativeadversarial-nets.pdf (last access: 26 May 2020), 2016.

Delanoë, J. and Hogan, R. J.: Combined CloudSat-CALIPSOMODIS retrievals of the properties of ice clouds, J. Geophys. Res., 115, D00H29, https://doi.org/10.1029/2009JD012346, 2010.

Del Guasta, M., Morandi, M., Stefanutti, L., Brechet, J., and Piquad, J.: One year of cloud lidar data from Dumont d'Urville (Antarctica): 1. General overview of geometrical and optical properties, J. Geophys. Res., 98, 18575-18587, https://doi.org/10.1029/93JD01476, 1993.

Donahue, J. and Simonyan, K.: Large Scale Adversarial Representation Learning, arXiv [preprint], arXiv1907.02544, 5 November 2019.

Donahue, J., Krähenbühl, P., and Darrell, T.: Adversarial Feature Learning, arXiv [preprint], arXiv1605.09782, 3 April 2017.

Elsaesser, G. S., Del Genio, A. D., Jiang, J. H., and van Lier-Walqui, M.: An Improved Convective Ice Parameterization for the NASA GISS Global Climate Model and Impacts on Cloud Ice Simulation, J. Climate, 30, 317-336, https://doi.org/10.1175/JCLI-D16-0346.1, 2017.

Feind, R. E.: Thunderstorm In Situ Measurements from the Armored T-28 Aircraft: Comparison of Measurements from Two Liquid Water Instruments and Classification of 2D Probe Hydrometeor Images, PhD thesis, South Dakota School of Mines and Technology, Rapid City, South Dakota, USA, 2006.

Flato, G., Marotzke, J., Abiodun, B., Braconnot, P., Chou, S. C., Collins, W., Cox, P., Driouech, F., Emori, S., Eyring, V., Forest, C., Gleckler, P., Guilyardi, E., Jakob, C., Kattsov, V., Reason, C., and Rummukainen, M.: Evaluation of Climate Models, in: Climate Change 2013: The Physical Science Basis. Contribution of Working Group I to the Fifth Assessment Report of the Intergovernmental Panel on Climate Change, edited by: Stocker, T. F., Qin, D., Plattner, G.-K., Tignor, M., Allen, S. K., Boschung, J., Nauels, A., Xia, Y., Bex, V., and Midgley, P. M., Cambridge University Press, Cambridge, UK, chap. 9, 741-866, 2013.

Fukunaga, K.: Parameter Estimation, in: Introduction to Statistical Pattern Recognition, Academic Press, Boston, Massachusetts, USA, 2nd edn., chap. 5, 181-253, https://doi.org/10.1016/B9780-08-047865-4.50011-9, 1990.

Garrett, T. J., Fallgatter, C., Shkurko, K., and Howlett, D.: Fall speed measurement and high-resolution multi-angle photography of hydrometeors in free fall, Atmos. Meas. Tech., 5, 26252633, https://doi.org/10.5194/amt-5-2625-2012, 2012.
Gaustad, K., Shkurko, K., and Garrett, T.: ARM: Multi-angle Snowflake Camera, analysis per particle (images and their aggregation), US Department of Energy Atmospheric Radiation Measurement Program, https://doi.org/10.5439/1350635, 2015.

Genthon, C., Berne, A., Grazioli, J., Durán Alarcón, C., Praz, C., and Boudevillain, B.: Precipitation at Dumont d'Urville, Adélie Land, East Antarctica: the APRES3 field campaigns dataset, Earth Syst. Sci. Data, 10, 1605-1612, https://doi.org/10.5194/essd-10-1605-2018, 2018.

Goodfellow, I., Pouget-Abadie, J., Mirza, M., Xu, B., WardeFarley, D., Ozair, S., Courville, A., and Bengio, Y.: Generative Adversarial Nets, in: Advances in Neural Information Processing Systems 27, edited by: Ghahramani, Z., Welling, M., Cortes, C., Lawrence, N. D., and Weinberger, K. Q., Curran Associates, Inc., 2672-2680, available at: https://papers.nips.cc/ paper/5423-generative-adversarial-nets.pdf (last access: 26 May 2020), 2014.

Goodfellow, I., Bengio, Y., and Courville, A.: Deep Learning, MIT Press, Cambridge, Massachusetts, USA, available at: https: //www.deeplearningbook.org/ (last access: 26 May 2020), 2016.

Grazioli, J., Genthon, C., Boudevillain, B., Duran-Alarcon, C., Del Guasta, M., Madeleine, J.-B., and Berne, A.: Measurements of precipitation in Dumont d'Urville, Adélie Land, East Antarctica, The Cryosphere, 11, 1797-1811, https://doi.org/10.5194/tc-111797-2017, 2017.

Gulrajani, I., Ahmed, F., Arjovsky, M., Dumoulin, V., and Courville, A. C.: Improved Training of Wasserstein GANs, in: Advances in Neural Information Processing Systems 30, edited by: Guyon, I., Luxburg, U. V., Bengio, S., Wallach, H., Fergus, R., Vishwanathan, S., and Garnett, R., Curran Associates, Inc., 5767-5777, available at: https://papers.nips.cc/ paper/7159-improved-training-of-wasserstein-gans.pdf (last access: 26 May 2020), 2017.

Gultepe, I., Heymsfield, A. J., Field, P. R., and Axisa, D.: Ice-Phase Precipitation, Meteor. Mon., 58, 6.1-6.36, https://doi.org/10.1175/AMSMONOGRAPHS-D-16-0013.1, 2017.

He, K., Zhang, X., Ren, S., and Sun, J.: Deep Residual Learning for Image Recognition, in: The IEEE Conference on Computer Vision and Pattern Recognition (CVPR), Las Vegas, NV, USA, 27-30 June 2016, IEEE, 770-778, https://doi.org/10.1109/CVPR.2016.90, 2016.

Hicks, A. and Notaroš, B. M.: Method for Classification of Snowflakes Based on Images by a Multi-Angle Snowflake Camera Using Convolutional Neural Networks, J. Atmos. Ocean. Tech., 36, 2267-2282, https://doi.org/10.1175/JTECHD-19-0055.1, 2019.

Huang, X. and Belongie, S.: Arbitrary Style Transfer in RealTime With Adaptive Instance Normalization, in: The IEEE International Conference on Computer Vision (ICCV), Venice, Italy, 22-29 October 2017, IEEE, 1501-1510, https://doi.org/10.1109/ICCV.2017.167, 2017.

Ioffe, S. and Szegedy, C.: Batch normalization: Accelerating deep network training by reducing internal covariate shift, arXiv [preprint], arXiv1502.03167, 2 March 2015.

Jain, A. K., Murty, M. N., and Flynn, P. J.: Data Clustering: A Review, ACM Comput. Surv., 31, 264-323, https://doi.org/10.1145/331499.331504, 1999. 
Karras, T., Laine, S., and Aila, T.: A Style-Based Generator Architecture for Generative Adversarial Networks, in: The IEEE Conference on Computer Vision and Pattern Recognition (CVPR), Long Beach, California, USA, 16-20 June 2019, IEEE, 44014410, https://doi.org/10.1109/CVPR.2019.00453, 2019.

Kaufman, L. and Rousseeuw, P. J.: Finding Groups in Data: An Introduction to Cluster Analysis, John Wiley \& Sons, Hoboken, New Jersey, USA, 1990.

Kingma, D. P. and Ba, J.: Adam: A method for stochastic optimization, in: 3rd International Conference for Learning Representations, San Diego, California, USA, 7-9 May 2015.

Kuhn, T. and Vázquez-Martín, S.: Microphysical properties and fall speed measurements of snow ice crystals using the Dual Ice Crystal Imager (D-ICI), Atmos. Meas. Tech., 13, 1273-1285, https://doi.org/10.5194/amt-13-1273-2020, 2020.

Lamb, D. and Verlinde, J.: Physics and Chemistry of Clouds, Cambridge University Press, Cambridge, United Kingdom, https://doi.org/10.1017/CBO9780511976377, 2011.

LeCun, Y., Bengio, Y., and Hinton, G.: Deep Learning, Nature, 521, 436-444, https://doi.org/10.1038/nature14539, 2015.

Leinonen, J.: Snow-gan-classification, available at: https://github. com/jleinonen/snow-gan-classification (last access: 26 May 2020), 2019.

Leinonen, J., Lebsock, M. D., Tanelli, S., Sy, O. O., Dolan, B., Chase, R. J., Finlon, J. A., von Lerber, A., and Moisseev, D.: Retrieval of snowflake microphysical properties from multifrequency radar observations, Atmos. Meas. Tech., 11, 5471-5488, https://doi.org/10.5194/amt-11-5471-2018, 2018.

Leinonen, J., Guillaume, A., and Yuan, T.: Reconstruction of Cloud Vertical Structure With a Generative Adversarial Network, Geophys. Res. Lett., 46, 7035-7044, https://doi.org/10.1029/2019GL082532, 2019.

Lindqvist, H., Muinonen, K., Nousiainen, T., Um, J., McFarquhar, G. M., Haapanala, P., Makkonen, R., and Hakkarainen, H.: Ice-cloud particle habit classification using principal components, J. Geophys. Res., 117, D16206, https://doi.org/10.1029/2012JD017573, 2012.

Löffler-Mang, M. and Joss, J.: An Optical Disdrometer for Measuring Size and Velocity of Hydrometeors, J. Atmos. Ocean. Tech., 17, 130-139, https://doi.org/10.1175/15200426(2000)017<0130:AODFMS>2.0.CO;2, 2000.

Mace, G. and Benson, S.: Diagnosing Cloud Microphysical Process Information from Remote Sensing Measurements - A Feasibility Study Using Aircraft Data. Part I: Tropical Anvils Measured during TC4, J. Appl. Meteorol. Clim., 56, 633-649, https://doi.org/10.1175/JAMC-D-16-0083.1, 2017.

Miyato, T., Kataoka, T., Koyama, M., and Yoshida, Y.: Spectral Normalization for Generative Adversarial Networks, in: International Conference on Learning Representations, arXiv [preprint], arXiv1802.05957, 16 February 2018.

Molthan, A. L. and Colle, B. A.: Comparisons of Single- and Double-Moment Microphysics Schemes in the Simulation of a Synoptic-Scale Snowfall Event, Mon. Weather Rev., 140, 29823002, https://doi.org/10.1175/MWR-D-11-00292.1, 2012.

Morrison, H., Milbrandt, J. A., Bryan, G. H., Ikeda, K., Tessendorf, S. A., and Thompson, G.: Parameterization of Cloud Microphysics Based on the Prediction of Bulk Ice Particle Properties. Part II: Case Study Comparisons with Obser- vations and Other Schemes, J. Atmos. Sci., 72, 312-339, https://doi.org/10.1175/JAS-D-14-0066.1, 2015.

Nair, V. and Hinton, G. E.: Rectified linear units improve restricted Boltzmann machines, in: Proceedings of the 27th International Conference on International Conference on Machine Learning (ICML-10), Haifa, Israel, 21-24 June 2010, Omnipress, 807814, 2010.

Newman, A. J., Kucera, P. A., and Bliven, L. F.: Presenting the Snowflake Video Imager (SVI), J. Atmos. Ocean. Tech., 26, 167179, https://doi.org/10.1175/2008JTECHA1148.1, 2009.

Notaroš, B. M., Bringi, V. N., Kleinkort, C., Kennedy, P., Huang, G.-J., Thurai, M., Newman, A. J., Bang, W., and Lee, G.: Accurate Characterization of Winter Precipitation Using Multi-Angle Snowflake Camera, Visual Hull, Advanced Scattering Methods and Polarimetric Radar, Atmosphere, 7, 81, https://doi.org/10.3390/atmos7060081, 2016.

Park, T., Liu, M.-Y., Wang, T.-C., and Zhu, J.-Y.: Semantic Image Synthesis With Spatially-Adaptive Normalization, in: The IEEE Conference on Computer Vision and Pattern Recognition (CVPR), Long Beach, California, USA, 15-20 June 2019, IEEE, 2337-2346, https://doi.org/10.1109/CVPR.2019.00244, 2019.

Praz, C., Roulet, Y.-A., and Berne, A.: Solid hydrometeor classification and riming degree estimation from pictures collected with a Multi-Angle Snowflake Camera, Atmos. Meas. Tech., 10, 13351357, https://doi.org/10.5194/amt-10-1335-2017, 2017.

Rojas, R.: The Backpropagation Algorithm, in: Neural Networks: A Systematic Introduction, Springer, Berlin, Heidelberg, chap. 7, 149-182, https://doi.org/10.1007/978-3-642-61068-4_7, 1996.

Schaer, M., Praz, C., and Berne, A.: Identification of blowing snow particles in images from a Multi-Angle Snowflake Camera, The Cryosphere, 14, 367-384, https://doi.org/10.5194/tc-14367-2020, 2020.

Schönhuber, M., Lammer, G., and Randeu, W. L.: One decade of imaging precipitation measurement by $2 \mathrm{D}$-video-distrometer, Adv. Geosci., 10, 85-90, https://doi.org/10.5194/adgeo-10-852007, 2007.

Ulyanov, D., Vedaldi, A., and Lempitsky, V.: Instance Normalization: The Missing Ingredient for Fast Stylization, arXiv [preprint], arXiv:1607.08022, 6 November 2017a.

Ulyanov, D., Vedaldi, A., and Lempitsky, V.: It Takes (Only) Two: Adversarial Generator-Encoder Networks, arXiv [preprint], arXiv:1704.02304, 6 November 2017b.

Wang, Z., Bovik, A. C., Sheikh, H. R., and Simoncelli, E. P.: Image quality assessment: from error visibility to structural similarity, IEEE T. Image Process., 13, 600-612, https://doi.org/10.1109/TIP.2003.819861, 2004.

Wood, N. B., L'Ecuyer, T. S., Heymsfield, A. J., Stephens, G. L., Hudak, D. R., and Rodriguez, P.: Estimating snow microphysical properties using collocated multisensor observations, J. Geophys. Res.-Atmos., 119, 8941-8961, https://doi.org/10.1002/2013JD021303, 2014. 